\title{
Laparoscopic Partial or Subtotal Intersphincteric Resection for Lower Rectal Cancer
}

\author{
Dr. Pilli Kalyan Chakravarthy ${ }^{1}$, Dr. Pankaj kumar ${ }^{1}$, ZHANG Shouliang ${ }^{1}$, \\ LIN Jian-yang ${ }^{1}$, LIU Jun ${ }^{1}$,Wei Fa xiang ${ }^{1}$, CHEN Zhi-hong ${ }^{1}$. \\ ${ }^{1 .}$ Department of General Surgery, The Affiliated People's Hospital of Jiangsu University, Zhenjiang 212002 \\ Jiangsu province, China
}

\begin{abstract}
Objective to investigate the treatment probability of laparoscopic partial or subtotal intersphincteric resection for ultra-low rectal cancer. Clinical data of 14 patients with ultra-low rectal cancer who have underwent laparoscopic partial or subtotal intersphincteric resection between January 2013 and February 2014 in gastrointestinal surgery was collected. Clinical application effect of laparoscopic partial or subtotal intersphincteric resection for ultra-low rectal cancer was analyzed.Results: Among all the cases, nobody got peri-operative death, one patient got partial anastomotic crack a week after operation and another patient got 12 days postoperative anastomotic fistula, both of whom cured through conservative treatment. During the 654-month follow-up, there was 1 case of local recurrence after 6 months of operation. The anal functions of all patients turned out to be in Kirwan class 1 or 2 and 1 patient got anastomotic stenosis, who required regular expansion treatment.
\end{abstract}

Conclusion Laparoscopic partial or subtotal intersphincteric resection for ultra-low rectal cancer was a safe and possible operational method.

Keywords: Intersphincteric resection; Laparoscopic surgery; Rectal cancer.

\section{Introduction}

For the lower portion of the tumor from rectal cancer of the anal margin $<5 \mathrm{~cm}$, traditional upward after abdominoperineal resection (abdominoperineal resection,APR). This procedure proceeds in the left lower quadrant abdominal colostomy patients with a permanent colostomy, and cannot easily accept. In 1992 Braun et al[1].first reported by resection of internal sphincter (intersphincteric resection,ISR) in the treatment of tumor position of Ultralow rectal cancer of the anal $5 \mathrm{~cm}$ in 2012 Ireland by doctor Martin[2]. System reviews the 14 units were reported, 1289 admits patients with intersphincteric resection for low rectal cancer information, where resection rate(RO) of $97 \%$, operative mortality is $0.8 \%, 5$-year survival rate is an average of $86.3 \%, 5$ year disease-free survival rate was $78.6 \%$, anal function full scale is $51.2 \%$. Yamada [3]. According to the different distance between cancer and anal edge, ISR can be classified as completely internal sphincter resection (Total ISR), Subtotal resection of internal sphincter (Subtotal ISR) and partial internal sphincter resection (Partial ISR). Total of ISR in postoperative patients with defecation control the Subtotal and Partial ISR, so Total ISR indications must be used with attention. Laparoscopic total mesorectal excision technique (Total Mesorectal excision,TME). Technology is already established in major hospitals. Collect now from January 2013 to February 2014 in gastrointestinal surgery laparoscopic partial or subtotal intersphincteric resection in the treatment of 14 cases of ultralow rectal cancer and clinical data, analysis of laparoscopic partial or subtotal resection of internal sphincter on the clinical effect of treatment of super-low rectal cancer.

\section{General Information:}

\section{Materials and methods}

From January 2013 to February 2014 time period, in the gastrointestinal surgical laparoscopic partial or sub total resection sphincter ultra low colorectal cancer 14 cases, including 6 males, 8 females,aged 32 to 74 years old,average 55.2years old. 9 cases of tumor type and the other five cases are ulcerated. All the depth of tumor invasion, preoperative $\mathrm{CT}$ and MRI confirmed $\leq \mathrm{T} 2$, sphincter and pelvic floor muscles were not invaded and at the muscular and rectal cancer the surrounding tissue in the image there is a clear dividing line; regional lymph node metastasis, preoperative CT and MRI found one case stage wasN1a, 13,N0.All patients were not found any distant metastasis and histological classification found two cases of villous adenoma recurrence and local cancer, 12 cases for the-well-differentiated adeno carcinoma.Under the tumor edge from the anal margin 2.5 $4.5 \mathrm{~cm}$, the average tumor diameter is $4.1 \mathrm{~cm}$. No bowel dysfunction in all patients before surgery.

\section{Operative Methods:}

Under general anesthesia Head and foot-high lithotomy, right hip joint bending $\left(10 \sim 15^{\circ}\right)$ less than the left. Establishment of artificial pneumoperitoneum observation hole of each operation holes. First we were cut 
the root of the inferior mesenteric artery on peritoneal surfaces then cleaning NO.253 lymph nodes and the root transaction of the blood vessels. The blood vessels from breaking the sigmoid colon of lymph node dissection NO.252, were using the TME technique(Total mesorectal excision, TME) free rectumits mesentery of the levatorani muscle level to separation between $1 \sim 2 \mathrm{~cm}$ down the intestinal wall or sphincter ring and outside the internal

sphincter.while preserving the pelvic autonomic. Followed by anal surgery. Anal four fingers in the perianal 2point, 5:00, 7:00, 10:00, respectively anus skin traction sutures and supplemented Park retractors revealed the surgical field the margin of at least $1 \sim 2 \mathrm{~cm}$ (T1period, $>1 \mathrm{~cm}$, thanT2 stage, $>2 \mathrm{~cm}$ ) from the tumor under perpendicular ano rectal cut the long axis of the dentate line or anal skin incision before the scheduled tangent or epinephrine injection diluted $(1: 2,000,000)$ to prevent bleeding. Purse-string suture to close the proximal end of the anal canal. Put Drain the separation between internal and external sphincter upto meeting with the abdominal

surgery group separated by the rear wall side walls front wall of the order the rectum and internal sphincter times en bloc resection of all or part of the far margin lateral margin send quick frozen sections check to make sure no residual cancer cells. Finally pulled from the anus to the sigmoid colon, sigmoid and anal anastomosis (Figure 1), if necessaryto ensure the free splenic flexure tension-free anastomosis placed inside the anus and anal canal decompression and drainage

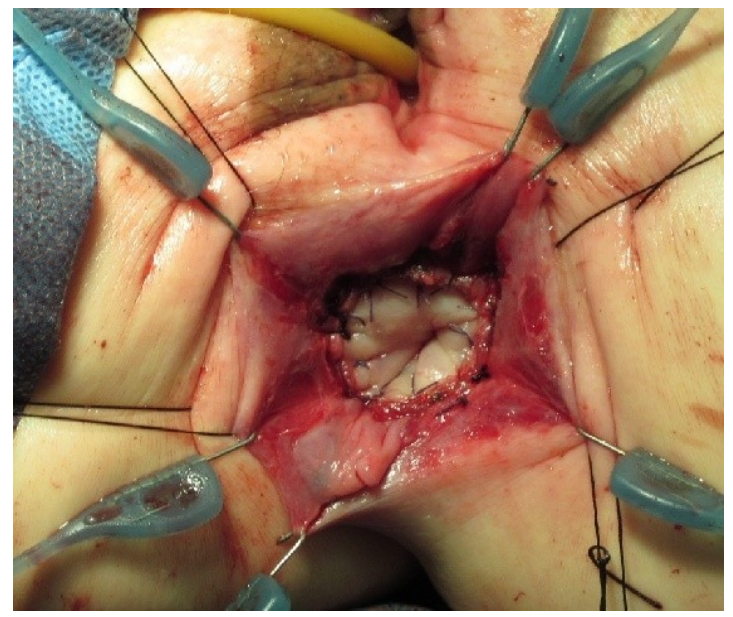

(Figure) 1.The sigmoid colon-anal anastomosis

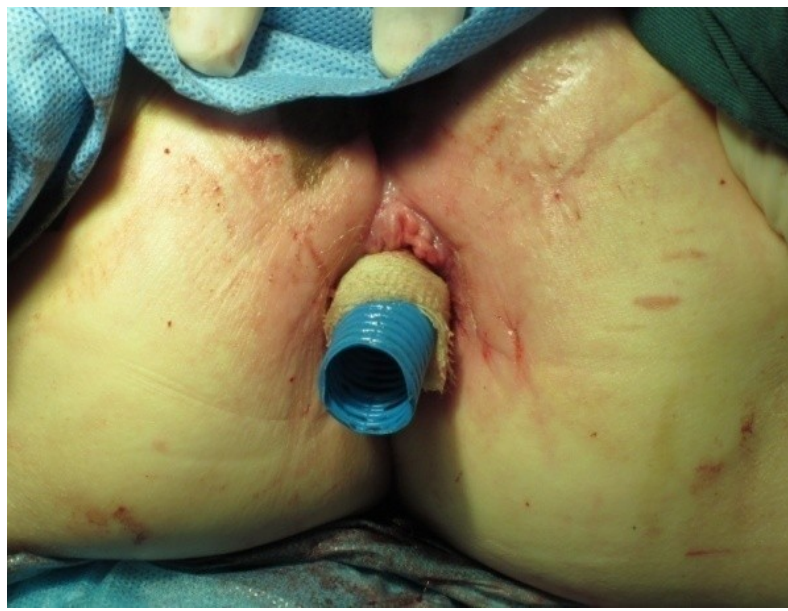

(Figure)2. Placed inside the anus and anal canal Decompression and drainage

\section{Results}

\section{A .PerioperativeCase:}

The 8 cases part of ISR and6 routine patients with subtotal ISR. 14 patients were operated smoothly, without conversion to laparotomy was no perioperative death. After 1 week of anastomotic partial dehiscence manure, cured by conservative and 1 fever cases 12days and anastomotic leakage fecal drainage tube from leads cured by local irrigation + drainage + support. 2 cases of postoperative ISR + end Ileostomy and no complications such as fistula, 3-4 months down, Ileostomy is satisfied. 3.5 5.0 h operation time intra-operative bleeding 100 250 $\mathrm{ml}, 2 \sim 4 \mathrm{~d}$ anus after anal screwed up external gas-sealed plastic bag.

\section{B . Pathological:}

The final diagnosis in all patients (Final findings), 5patients were fT1N0M0, 7cases fT2N0M0, 1cases fT2N1aM0, 1cases fT2N1bM0. Histological classification, 14 cases were in-well-differentiated adenocarcinoma.

\section{C .ThePostoperative Follow-up:}

Follw-up examinations include: blood CEA (Carcinoembryonic antigen, CEA) and the CA199, chest $x$ ray, CT and MRI of the abdomen. 2 cases of 4.5 years 4 cases followed up for 3 years, 5 cases were followed up for 2 years, 2 cases were followed up for 1 year and a half, 1 case was followed up for half a year,. 1 T2N0 and 2 $\mathrm{N}+$ patients with FOLFOX6 3 5 a course of adjuvant chemotherapy after surgery. 1 patients with T2N1b on postoperative local recurrence was discovered in June, another APR surgery, survival and other follow-up examination in patients with no local recurrence and distant metastasis. Function band by Kirwan[4]. Evaluation level normal, level 2: unable to control exhaust, level 3 occasional small manure, 4: manure frequently, 5: fecal incontinence. All patients in the postoperative anal function restored within 6 months to Kirwan classification 1-2. 1 cases of anastomotic stenosis in the patients, need to be extended on a regular basis. 


\section{Discussion}

Surgical treatment of cancer is currently hope to achieve two purposes, one is to cure cancer,the second is to maintain a normal quality of life,avoiding defecation,urination, and sexual dysfunction. The lower edge of the tumor from the anal margin $<5 \mathrm{~cm}$ tumor, APR cure cancer surgery can only meet the purpose. ISR low colorectal cancer surgery is reserved sphincter function limits the surgery,but people are still concerned about whether this surgery will lead to disorders of high local recurrence and anal sphincter function. Recently,Yamada et al [3].Reported 35 cases, of T1 colorectal cancer, far margin of at least 1 cm, T2and T3 of colorectal cancer, distantmargins of at least $2 \mathrm{~cm}$,postoperative follow-up14-48months, no case of local recurrence, after December anal sphincter function were satisfactory. Portier et al [5] .Reported 173 cases and 105 cases of ISR ultra low colo-anal anastomosis surgery (ultra-low coloanal anastomosis, CAA) effect, found the two -5-year local recurrence rate and patient survival rates were not significantly differences Martin [2]. Systematically reviewed the 14 units were reported, 1289 to accept Subtotal, Partial and Total ISR low colorectal cancer surgery patient information, R0 resection rate was $97 \%$, the operative mortality was $0.8 \%$, the average rate of local recurrence $6.7 \%$, the average five-year survival rate was $86.3 \%$, the proportion of anal function was $51.2 \%$ complete recovery. After the summary of the general opinion is far from the edge of the cutting edge at least under the tumor $1 \mathrm{~cm}$ needs to be done if the tumor invasion anal Total ISR surgery. If the tumor invasion levator ani puborectalis and or externa lanal sphincter then APR surgery needs to be done. If the tumor with distant metastasis or poorly differentiated type is not suitable for ISR surgery. Our disposal standards are: lower edge of the tumor from the anal margin $<5 \mathrm{~cm}$; local infiltration confined within the intestinal wall or straight sphincter; histological grade high-differentiation. Standards are not suitable for the ISR: tumor invasion of the levator ani, puborectalis and or anal sphincter; existing bowel dysfunction. Far away from the tumor margin lower edge of at least $1 \sim 2 \mathrm{~cm}$ (T1period,> 1 cm; thanT2 stage, $>2 \mathrm{~cm}$ ). Results 14 cases of local recurrence in one case, all patients within 6 months after anal function restored to Kirwan grade 1-2level. Laparoscopic TME+ISR for ultra low rectal cancer therapy efficacy and superiority has increasingly been reported and concerns [6-9]. Complete repair of endoscopic TME, external sphincter ring and the intestinal wall or even further down the separation between internal sphincter $1 \sim 2 \mathrm{~cm}$, coupled with accurate separation between internal and external sphincter is the key to operation. Our experience is laparoscopic vision free throughout the pelvic floor has an advantage in the rectum .ISR laparoscopic rectum from anal pulledout, could be discharged from abdominal incision pain from anal dragged out of the blood supply of the sigmoid colon, related to healing of anastomosis. If the blood supply to the deviation, the older ( $>70$ years), the best end Ileostomy. The two cases anastomotic fistula and local split, possibly related to this.

\section{References}

[1] Braun J, Treutner KH, Winkeltau G, Heidenreich U, Lerch MM andSchumpelick V. Results of intersphincteric resection of the rectum with direct coloanal anastomosis for rectal carcinoma. Am J Surg 1992;163(4): 407-412.

[2] MartinST, Heneghan HM and Winter DC. Systematic review of outcomes after intersphincteric resection for low rectal cancer. Br J Surg 2012; 99(5): 603-612.

[3] Yamada K, Ogata S, Saiki Y, Fukunaga M, Tsuji Y and Takano M. Functional results of intersphincteric resection for low rectal cancer. Br J Surg 2007; 94(10): 1272-1277.

[4] Rullier E, Zerbib F, Laurent C, Bonnel C, Caudry M, Saric J andParneix M. Intersphincteric resection with excision of internal anal sphincter for conservative treatment of very low rectal cancer. Dis Colon Rectum 1999; 42(9):1168-1175.

[5] Portier G, Ghouti L, Kirzin S, Guimbaud R, Rives M and Lazorthes F. Oncological outcome of ultra-low coloanal anastomosis with and without intersphincteric resection for low rectal adenocarcinoma. Br J Surg2007; 94(3): 341-345.

[6] Orsenigo E, Di Palo S, Vignali A andStaudacher C. Laparoscopic intersphincteric resection for low rectal cancer. SurgOncol 2007; 16(Suppl 1): S117-S120.

[7] Lim SW, Huh JW, Kim YJ andKim HR. Laparoscopic intersphincteric resection for low rectal cancer. World Journal of Surgery $2011 ; 35(12): 2811-2817$.

[8] Hamada M, Matsumura T, Matsumoto T, Teraishi F, Ozaki K, Nakamura T, Fukui Y, Nishioka Y, Taniki T andHorimi T. Advantages of the laparoscopic approach for intersphincteric resection. SurgEndosc 2011; 25(5):1661-1663.

[9] Zhou Zhenxu, ZhengXiaofeng, Song Huayu, Jiang Fei Zhao, Wang Zhe, Zuozhigui, CaiHuajie and RuanXiaojiao. Laparoscopic total mesorectal excision combined with internal anal sphincter resection of Ultralow rectal cancer. Chinese Journal of gastrointestinal surgery $2011 ; 14(6)$ : 440-442. 